\title{
Attachment distribution of pectoral muscle origins identified in dual-plane breast implant insertion
}

\author{
Su Bong Nam ${ }^{1}$, Kyung Ho Song ${ }^{2}$, Jung Yeol Seo ${ }^{1}$, June Seok Choi ${ }^{1}$, Tae Seo Park ${ }^{1}$, Jae Woo Lee ${ }^{1}$, \\ Ju Hyung Kim ${ }^{1}$, Min Wook Kim ${ }^{1}$, Hyun Yeol Kim³ ${ }^{3}$ Yun Ju Jung ${ }^{3}$, Choongrak Kim ${ }^{4}$ \\ ${ }^{1}$ Department of Plastic and Reconstructive Surgery, Pusan National University School of Medicine, Yangsan; ${ }^{2}$ Four Seasons Plastic Surgery \\ Clinic, Busan; ${ }^{3}$ Breast Surgery Center, Pusan National University Yangsan Hospital, Yangsan; ${ }^{4}$ Department of Statistics, Pusan National \\ University, Busan, Korea
}

Background Implant-based dual-plane augmentation mammoplasty requires accurate separation of the pectoralis major muscle (PMM) at its origins. The authors identified the PMM origins during breast reconstruction surgery with the goal of providing additional information on subpectoral implant insertion for reconstructive or aesthetic purposes.

Methods This study was conducted on 67 patients who underwent breast reconstruction surgery at the breast center of our hospital between November 2016 and June 2018. In total, 34 left and 39 right hemithoraces were examined. The left and right hemithoraces were each divided into 15 zones to determine the percentage of PMM attachments in each zone. The distribution of PMM origins in each zone was examined to identify any statistically significant differences.

Results There were no statistically significant differences in the origins of the PMM between the right and left hemithoraces. The percentage of attachments increased moving from the fourth to the sixth rib and from the lateral to the medial aspect.

Conclusions The anatomical findings of this study could be used as a reference for accurate dissection of the origins of the PMM for the preparation of the subpectoral pocket for subpectoral implant placement.

Keywords Pectoralis muscle / Anatomy / Breast

\author{
Correspondence: Min Wook Kim \\ Department of Plastic and \\ Reconstructive Surgery, Pusan \\ National University School of \\ Medicine, 49 Busandaehak-ro, \\ Mulgeum-eup, Yangsan 50612, Korea \\ Tel: +82-55-360-2571 \\ Fax: +82-55-360-2158 \\ E-mail: minugi0619@hanmail.net
}

\begin{abstract}
This study was supported by a grant from the clinical research fund of Pusan National University Yangsan Hospital in 2016.

This article was presented at the 7th Research and Reconstruction Forum (REtR), on April 20-21, 2017, in Daejeon, Korea.
\end{abstract}

Received: July 16, $2019 \bullet$ Revised: December 17, $2019 \bullet$ Accepted: January 8, 2020

pISSN: 2234-6163 • elSSN: 2234-6171 • https://doi.org/10.5999/aps.2019.00906• Arch Plast Surg 2020;47:160-164

\section{INTRODUCTION}

In implant-based breast augmentation or reconstruction mammoplasty, an implant may be placed under glandular tissue, fascia, or the pectoralis major muscle (PMM) according to the surgeon's experience or the shape of the breast [1]. Dual-plane augmentation mammoplasty, first introduced by Tebbetts [2] in 2001, utilizes the space under the PMM for the superior portion of the implant while placing its inferior part under deep fatty tissue or glandular tissue. This surgical technique has yielded positive results, as it reproduces the natural shape of the breast, has a low complication rate, and promotes fast recovery. The precision of this technique depends on the positioning of the implant within the breast, which in turn requires accurate separation of the PMM at its origins on the ribs and cartilages. Incomplete removal of the origins of the PMM can distort the position of the 
implant postoperatively, mimic a contracture deformity, and cause a depression deformity between the implant and breast tissue during PMM contraction [3]. In contrast to numerous studies on the origins of the PMM on the sternum [4], few studies have been conducted on the origins of the PMM on the ribs and cartilages, as anatomical studies using breast augmentation patients or cadavers are difficult to perform due to practical limitations. Therefore, this study was conducted to directly verify the origins of the PMM through extended incisions made during immediate breast reconstruction after total mastectomy for breast cancer. The goal of this study was to provide additional information on subpectoral implant insertion for reconstructive or aesthetic purposes.

\section{METHODS}

\section{Study subjects}

The study was conducted on 67 patients (73 hemithoraces) who underwent breast reconstruction surgery using either permanent implants only or extended latissimus dorsi muscle flaps with permanent implants at the breast center of our hospital during the 20-month period between November 2016 and June 2018. In total, 34 left and 39 right hemithoraces were examined, and the average age of the patients was 49.1 years.

\section{Surgical methods}

After skin- and nipple-sparing mastectomy above the PMM in breast surgery, most of the PMM is exposed, and the sixth rib and costal cartilage of the inframammary fold come into contact. Based on this, the incision site of the PMM was marked, and the location of the pocket for dual-plane type II augmentation was confirmed. A careful incision was initiated along the lateral margin of the PMM to prevent damage to the serratus anterior muscle. When muscle fibers attached to ribs or costal cartilages on the undersurface of the PMM were identified, they were separated using bipolar coagulators. In the intercostal space, the muscles, including the blood vessels, are attached to their origins. These were separated carefully with hemostasis. The PMM attached to the sixth rib and the costal cartilage was separated to the medial aspect. Then, the separation was continued upwards, with care not to damage the internal mammary vessels by touching the lateral end of the sternal border. All PMM separation proceeded from the lateral to the medial aspect and from the inferior to the superior aspect to the fourth rib and costal cartilage. In the lateral margin of the PMM, the serratus anterior muscles and the pectoralis minor muscles under the PMM were separated without damage.

From the center of the costal cartilages, the inner surface was designated as zone $\mathrm{A}$ (lateral to the branching point of the internal mammary artery), the outer surface as zone $B$, and the space between the costochondral junction and the midclavicular line as zone $\mathrm{C}$. The proportions of these zones where attachments of the PMM were present were determined (Fig. 1).

\section{Statistical analysis}

All statistical analyses of the obtained values were performed using R Statistics version 3.5.3 (R Project for Statistical Computing, Vienna, Austria). The equality of two proportions test was performed, using the two-sample Z-test to evaluate the statistical significance $(\mathrm{P}<0.05)$ of the difference between the right and left hemithoraces. The one-sided Jonckheere-Terpstra test $\left(\mathrm{H}_{0}: \mathrm{p}_{1}=\mathrm{p}_{2}=\mathrm{p}_{3}=\mathrm{p}_{4}=\mathrm{p}_{5}\right.$ vs. $\left.\mathrm{H}_{1}: \mathrm{p}_{1}<\mathrm{p}_{2}<\mathrm{p}_{3}<\mathrm{p}_{4}<\mathrm{p}_{5}\right)$ was utilized to evaluate the statistical significance $(\mathrm{P}<0.05)$ of the differences in the distribution of attachments of the PMM obtained from the fourth to the sixth rib. In the same way, the one-sided Jonckheere-Terpstra test $\left(\mathrm{H}_{0}: \mu_{\mathrm{A}}=\mu_{\mathrm{B}}=\mu_{\mathrm{C}}\right.$ vs. $\left.\mathrm{H}_{1}: \mu_{\mathrm{A}}>\mu_{\mathrm{B}}>\mu_{\mathrm{C}}\right)$ was utilized to evaluate the statistical significance $(\mathrm{P}<0.05)$ of the differences in the distribution of attachments of the PMM among zones $\mathrm{A}, \mathrm{B}$, and $\mathrm{C}$.

\section{RESULTS}

In the patients who underwent immediate breast reconstruction using either an extended latissimus dorsi flap with a permanent

\section{Fig. 1. Division of zones}

Zone A from the branching point of the internal mammary artery to the center of the costal cartilage. Zone B from the center of the costal cartilage to costochondral junction. Zone $\mathrm{C}$ from costochondral junction to the midclavicular line.

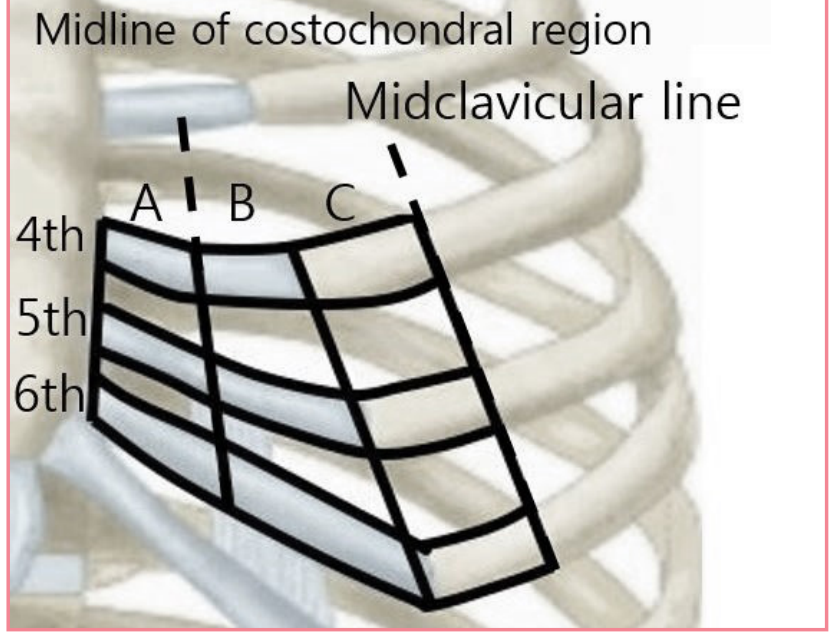


implant or a permanent implant only at the breast center of our hospital, the origins of the PMM on the ribs and cartilage in the right and left hemithoraces were verified during surgery.

In the 67 patients ( 73 hemithoraces), $71 \%, 59 \%$, and $53 \%$ of zones $\mathrm{A}, \mathrm{B}$, and $\mathrm{C}$ of the left fourth rib contained attachments, respectively. Using zone $\mathrm{C}$ as a reference, on the left fourth rib, fourth intercostal region, fifth rib, fifth intercostal region, and sixth rib, attachments were present in 53\%, 65\%, 88\%, 82\%, and $97 \%$ of the zones, respectively. On the right fourth rib, $87 \%$, $85 \%$, and $79 \%$ of zones A, B, and C contained attachments, respectively. Using zone $\mathrm{C}$ as a reference, on the right fourth rib, fourth intercostal region, fifth rib, fifth intercostal region, and sixth rib, attachments were present in 79\%, 69\%, 79\%, 87\%, and $92 \%$ of the zones, respectively (Table 1 ).

A comparison between the right and left fourth ribs revealed a significant difference $(\mathrm{P}<0.01)$. However, comparisons of the fourth intercostal region, the fifth rib, the fifth intercostal region, and the sixth rib showed no significant difference between the right and left hemithoraces, with P-values of 0.23, >0.99, 0.99, and 0.45 , respectively (Table 2 ).

A statistically significant increase was observed in the percentage of attachments in the left hemithorax when descending from the fourth to the sixth rib $(\mathrm{P}=0.01)$. Likewise, a statistically significant increase in the percentage of attachments was observed

\section{Table 1. Presence of origins of both pectoralis major muscles}

\begin{tabular}{|lccc|}
\hline Location & A zone (\%) & B zone (\%) & C zone (\%) \\
\hline Left hemithorax & & & \\
Fourth rib & 71 & 59 & 53 \\
Fourth intercostal region & 79 & 76 & 65 \\
Fifth rib & 97 & 94 & 88 \\
Fifth intercostal region & 100 & 100 & 82 \\
Sixth rib & 100 & 100 & 97 \\
Right hemithorax & & & \\
Fourth rib & 87 & 85 & 79 \\
Fourth intercostal region & 87 & 87 & 69 \\
Fifth rib & 97 & 100 & 79 \\
Fifth intercostal region & 95 & 97 & 87 \\
Sixth rib & 100 & 97 & 92 \\
\hline
\end{tabular}

Table 2. Comparative analysis between the left and right hemithoraces

\begin{tabular}{|lcccc|}
\hline \multirow{2}{*}{ Location } & \multicolumn{4}{c|}{ P-value } \\
\cline { 2 - 5 } & $\mathrm{A}+\mathrm{B}+\mathrm{C}$ zone & $\mathrm{A}$ zone & $\mathrm{B}$ zone & $\mathrm{C}$ zone \\
\hline Fourth rib & $<0.01$ & 0.14 & 0.03 & 0.03 \\
Fourth intercostal region & 0.23 & 0.56 & 0.38 & 0.87 \\
Fifth rib & $>0.99$ & $>0.99$ & 0.41 & 0.49 \\
Fifth intercostal region & 0.99 & 0.54 & $>0.99$ & 0.80 \\
Sixth rib & 0.45 & $>0.99$ & $>0.99$ & 0.71 \\
\hline
\end{tabular}

in the right hemithorax when descending from the fourth to the sixth rib $(\mathrm{P}=0.04)$ (Table 3$)$.

In the left hemithorax, the percentage of attachments decreased moving from the medial to the lateral aspect (from zone A to $B$ and from zone $B$ to $C$ ), but without statistical significance $(P=0.10)$. In the right hemithorax, the percentage of attachments also decreased from zone $\mathrm{A}$ to $\mathrm{B}$ and from zone $\mathrm{B}$ to $\mathrm{C}$, but in contrast with the left side, this trend was statistically significant $(\mathrm{P}=0.02)$ (Table 4).

\section{DISCUSSION}

Major progress has been made in the field of breast augmentation since Czerny's first report on the augmentation of a breast defect using a lipoma in 1895 [5], followed by Cronin and Gerow's first use of silicone gel as an implant material for breast augmentation [6]. The use of implants for breast augmentation or immediate reconstruction after mastectomy is fairly common in Korean women due to their small breast size compared to women in Western populations. In most cases of implant-based breast augmentation or reconstruction, submuscular placement under the PMM offers the most ideal plane. This is because it can prevent bulging and show-through of the implants in women with insufficient parenchymal tissue by reinforcing the soft

Table 3. Statistical significance analysis of the differences in the distribution of attachments of the pectoralis major muscle from the fourth to the sixth rib

\begin{tabular}{ll|}
\hline Hypothesis & P-value \\
\hline Left hemithorax & 0.01 \\
$H_{0}: p_{1}=p_{2}=p_{3}=p_{4}=p_{5}$ & \\
$H_{1}: p_{1}<p_{2}<p_{3}<p_{4}<p_{5}$ & 0.04 \\
Right hemithorax & \\
$H_{0}: p_{1}=p_{2}=p_{3}=p_{4}=p_{5}$ & \\
$H_{1}: p_{1}<p_{2}<p_{3}<p_{4}<p_{5}$ & \\
\hline$H_{0}$, null hypothesis; $H_{1}$, alternative hypothesis; $p_{1}$, fourth rib; $p_{2}$, fourth intercostal \\
region; $p_{3}$, fifth rib; $p_{4}$, fifth intercostal region; $p_{5}$, sixth rib.
\end{tabular}

Table 4. Statistical significance analysis of the differences in the distribution of attachments of the pectoralis major muscle among zones $A, B$, and $C$

\begin{tabular}{|lc|}
\hline Hypothesis & P-value \\
\hline Left hemithorax & 0.10 \\
$H_{0}: \mu_{A}=\mu_{B}=\mu_{C}$ & \\
$H_{1}: \mu_{A}>\mu_{B}>\mu_{C}$ & \\
Right hemithorax & 0.02 \\
$H_{0}: \mu_{A}=\mu_{B}=\mu_{C}$ & \\
$H_{1}: \mu_{A}>\mu_{B}>\mu_{C}$ & \\
\hline$H_{0}$, null hypothesis; $H_{1}$, alternative hypothesis; $\mu_{A}$, zone A; $\mu_{B}$, zone B; $\mu_{c}$, zone C.
\end{tabular}


tissues that cover the implants and because it leads to fewer cases of capsular contraction as a complication [7]. However, completely overlaying the implant with muscle can cause distortion or displacement of the implant during muscle movement, and it can also complicate control of the volume of the upper breast and interfere with precise manipulation of the inframammary folds [4]. Recently, the dual-plane technique, in which partial resection of the origins under the PMM allows the superior part of the implant to be placed under the muscle and the inferior part under the mammary glands, was introduced to resolve such issues [2].

Because the results of dual-plane breast augmentation strongly depend on precise detachment of the origins of the PMM, a full understanding of the anatomical landmarks of the PMM is important. Anatomically, the PMM is largely divided into the clavicular head and the sternocostal head, the latter of which originates from the manubrium, sternal body, external oblique abdominal muscles, upper aponeurosis of the rectus abdominis, and the second through sixth costal cartilages and inserts into the intertubercular groove of the humerus.

In order to insert a breast implant into the subpectoral pocket using the dual-plane method, some of the origins on the ribs and cartilages must be separated. Despite the firm attachment of the PMM at its origins near the sternal border and the branching points of the internal mammary artery, the distribution of its attachments in the costochondral junction, intercostal spaces, and fourth through sixth costal cartilages is poorly understood. Accurate separation of the lateral PMM from the ribs and cartilages is of particular importance for forming subpectoral pockets, but insufficient information regarding this issue is available.

In a cadaveric study, Anson et al. [8] reported that the costal origin fibers of the pectoralis major originate from the second rib to the fifth rib. In our breast intraoperative study, however, in most patients, we found an origin of the PMM on the sixth rib. In particular, the sixth rib is where the inframammary fold line of the breast is present in women, which is considered important when separating the PMM to make a dual plane for inserting a breast implant. Sanchez et al. [9] reported that an attenuated or shortened medial PMM origin could promote over-release and medial implant malposition in repose. Abnormally abundant costal origins of the PMM could conceivably result in incomplete release of the inferior pectoralis border, leading to superior implant malposition. Conversely, over-release of the medial pectoralis major can also cause pectoralis major "window shading" and abnormal inferolateral implant movement when the muscle is contracted $[10,11]$.

The degree of attachment of the PMM was found to be thinner toward the top and closer to the lateral margin of the PMM.
Understanding the anatomy and distribution of the origins of the PMM on the ribs and cartilages during subpectoral dissection through a transaxillary approach is crucial and can be of tremendous help for less-experienced surgeons. Furthermore, the high density of blood vessels near the PMM origins means that the distribution of these origins is important to understand in order to perform precise hemostasis during detachments, to reduce the incidence of hematoma, and ultimately to prevent further surgical complications.

This study found no statistically significant differences in the origins of the PMM between the right and left hemithoraces, except in the zone of the fourth rib. In real-world clinical settings, no significant difference between the right and left hemithoraces should be expected. On the left, the percentage of attachments increased moving from the fourth to the sixth rib and from the lateral to the medial aspect. A similar trend was observed on the right side. Statistical analyses of these observations exhibited significant differences except for zones A, B, and $\mathrm{C}$ on the left side, where the difference in the percentage of attachments between the lateral and medial aspects showed no statistical significance $(P=0.10)$. However, this may have resulted from the small sample size of the study; we predict that the difference in the percentage of attachments in zones $\mathrm{A}, \mathrm{B}$, and $\mathrm{C}$ on the left side would exhibit a statistically significant difference, as on the right, if the sample size were increased. Understanding the distribution of the origins of the PMM facilitates precise dissection of the muscle during dual-plane augmentation mammoplasty and may help reduce surgical complications, such as postoperative deformities.

Spear et al. [1] stated that the greatest difference between implant placement in the subpectoral plane and in the dual plane is the subglandular dissection above the inferior border of the PMM. As confirmed by this study, the importance of dissection near the inferior border of the PMM, represented by the sixth rib and zone $\mathrm{C}$, must be recognized because the majority of the zone around the sixth rib contains PMM attachments, meaning that precision is required when forming the dual plane. Compared to the other zones of the PMM, zones A and B of the fourth rib are often overlooked because attachments are relatively sparse in these zones. Nonetheless, PMM attachments were identified in over $50 \%$ of those zones, a higher proportion than originally expected. This observation indicates that zones $\mathrm{A}$ and $\mathrm{B}$ of the fourth rib are also of significant clinical importance. Furthermore, although in total, zone $\mathrm{C}$ from the fourth rib to the sixth rib was shown to contain a comparatively sparse percentage of attachments, attachments were observed in over $50 \%$ of the region throughout the ribs, so it may be concluded that dissection in this zone also requires special attention. 
This study found a similar distribution of the origins of the PMM in the right and left hemithoraces, and origins were uniformly present from the medial to the lateral aspect of the sixth rib. Despite the relatively sparse percentage of attachments in the zone, the origins of the PMM also extended to the lateral aspect of the fourth rib. With the goal of precise preparation of the space for subpectoral implant placement, the anatomical findings of this study could be used as a reference for accurate dissection of the origins of the PMM.

\section{NOTES}

\section{Conflict of interest}

No potential conflict of interest relevant to this article was reported.

\section{Ethical approval}

The study was approved by the Institutional Review Board of Pusan National University Yangsan Hospital (IRB No. 05-2019087) and performed in accordance with the principles of the Declaration of Helsinki. Written informed consents were obtained.

\section{Author contribution}

Conceptualization: Nam SB, Song KH, Kim MW. Data curation: Nam SB, Kim MW. Formal analysis: Seo JY, Choi JS, Park TS, Kim C. Methodology: Kim MW, Kim C. Administration: Seo JY, Lee JW, Kim JH, Kim HY, Jung YJ. Visualization: Seo JY, Lee JW, Kim JH, Kim HY, Jung YJ. Writing - original draft and review \& editing: all authors. Approval of final manuscript: all authors.

\section{ORCID}

Su Bong Nam

Kyung Ho Song

Jung Yeol Seo

June Seok Choi

Tae Seo Park

Jae Woo Lee

Ju Hyung Kim https://orcid.org/0000-0002-9661-0879

https://orcid.org/0000-0002-3419-972X

https://orcid.org/0000-0003-0715-2229

https://orcid.org/0000-0003-2140-8550

https://orcid.org/0000-0002-1491-0828

https://orcid.org/0000-0002-0945-6966

https://orcid.org/0000-0002-4893-3761
Min Wook Kim https://orcid.org/0000-0001-8024-3608

Hyun Yeol Kim https://orcid.org/0000-0001-9008-1278

Yun Ju Jung https://orcid.org/0000-0002-1311-4950

Choongrak Kim https://orcid.org/0000-0002-3230-4477

\section{REFERENCES}

1. Spear SL, Bulan EJ, Venturi ML. Breast augmentation. Plast Reconstr Surg 2004; 114:73e-81e.

2. Tebbetts JB. Dual plane breast augmentation: optimizing implant-soft-tissue relationships in a wide range of breast types. Plast Reconstr Surg 2001;107:1255-72.

3. Hidalgo DA. Breast augmentation: choosing the optimal incision, implant, and pocket plane. Plast Reconstr Surg 2000; 105:2202-16.

4. Moore KL, Dalley AF. Clinically oriented anatomy. Philadelphia: Lippincott Williams \& Wilkins; 1999.

5. Czerny V. Plastischer Ersatz der Brustdrüse durch ein Lipom. Zentral Chir 1895;22:72.

6. Cronin TD, Gerow FJ. Augmentation mammoplasty: a new "natural feel" prosthesis. Transactions of the Third International Congress of Plastic Surgery; 1963 Oct 13-18; Amsterdam, the Netherlands. Excerpta Medica Foundation; p. 419.

7. Sohn BK, Chung YJ, Kim G, et al. Submuscular periareolar approach to augmentation mammoplasty in Korean women. Aesthetic Plast Surg 2000;24:455-60.

8. Anson BJ, Jamieson RW, O'conor VJ, et al. The pectoral muscles; an anatomical study of 400 body-halves. QBull Northwest Univ Med Sch 1953;27:211-8.

9. Sanchez ER, Sanchez R, Moliver C. Anatomic relationship of the pectoralis major and minor muscles: a cadaveric study. Aesthet Surg J 2014;34:258-63.

10. Lesavoy MA, Trussler AP, Dickinson BP. Difficulties with subpectoral augmentation mammaplasty and its correction: the role of subglandular site change in revision aesthetic breast surgery. Plast Reconstr Surg 2010;125:363-71.

11. Codner MA, Cohen AT, Hester TR. Complications in breast augmentation: prevention and correction. Clin Plast Surg 2001;28:587-95. 